\title{
Low molecular weight chitosan-coated silver nanoparticles are effective for the treatment of MRSA-infected wounds
}

This article was published in the following Dove Press journal:

International Journal of Nanomedicine

4 January 2017

Number of times this article has been viewed

\author{
Yinbo Peng' \\ Chenlu Song' \\ Chuanfeng Yang' \\ Qige Guo' \\ Min Yao ${ }^{1,2}$
}

'Department of Burns and Plastic Surgery, Shanghai Ninth People's Hospital, Institute of Traumatic Medicine, Shanghai Jiao Tong University School of Medicine, Shanghai, People's Republic of China; ${ }^{2}$ Department of Dermatology, Wellman Center for Photomedicine, Harvard Medical School, Massachusetts General Hospital, Boston, MA, USA
Correspondence: Min Yao Department of Burns and Plastic Surgery, Shanghai Ninth People's Hospital, Institute of Traumatic Medicine, Shanghai Jiao Tong University School of Medicine, 280 Mohe Road, Baoshan District, Shanghai 201900, People's Republic of China

Tel +86 2I 5669 II0I ext 6186

Fax +86 2161313990

Email my058@vip.sina.com
Abstract: Silver nanoparticles (AgNPs) are being widely applied as topical wound materials; however, accumulated deposition of silver in the liver, spleen, and other main organs may lead to organ damage and dysfunction. We report here that low molecular weight chitosan-coated silver nanoparticles (LMWC-AgNPs) are effective against methicillin-resistant Staphylococcus aureus (MRSA), have better biocompatibility, and have lower body absorption characteristics when compared with polyvinylpyrrolidone-coated silver nanoparticles (PVP-AgNPs) and silver nanoparticles without surface stabilizer (uncoated-AgNPs) in a dorsal MRSA wound infection mouse model. LMWC-AgNPs were synthesized by reducing silver nitrate with low molecular weight chitosan as a stabilizer and reducing agent, while PVP-AgNPs were synthesized using polyvinylpyrrolidone as a stabilizer and ethanol as a reducing agent. AgNPs with different surface stabilizers were identified by UV-visible absorption spectrometry, and particle size was determined by transmission electron microscopy. UV-visible absorption spectra of LMWCAgNPs, PVP-AgNPs and uncoated-AgNPs were similar and their sizes were in the range of 10-30 nm. In vitro experiments showed that the three types of AgNPs had similar MRSAkilling effects, with obvious effect at $4 \mu \mathrm{g} / \mathrm{mL}$ and $100 \%$ effect at $8 \mu \mathrm{g} / \mathrm{mL}$. Bacteriostatic annulus experiments also showed that all the three types of AgNPs had similar antibacterial inhibitory effect at $10 \mu \mathrm{g} / \mathrm{mL}$. Cell counting kit- 8 assay and Hoechst/propidium iodide (PI) staining showed that LMWC-AgNPs were significantly less toxic to human fibroblasts than PVP-AgNPs and uncoated-AgNPs. Treatment of mice with MRSA wound infection demonstrated that the three types of AgNPs effectively controlled MRSA wound infection and promoted wound healing. After continuous application for 14 days, LMWC-AgNPs-treated mice showed significantly reduced liver dysfunction as demonstrated by the reduced alanine aminotransferase and aspartate aminotransferase levels and liver deposition of silver, in comparison to mice treated with uncoated-AgNPs or PVP-AgNPs. Our results demonstrated that LMWC-AgNPs had good anti-MRSA effects, while harboring a better biocompatibility and lowering the body's absorption characteristics.

Keywords: silver nanoparticles, LMWC-AgNPs, MRSA, PVP-AgNPs, PVP, biocompatibility

\section{Introduction}

Silver nanoparticles (AgNPs) are increasingly being used as various types of external trauma materials due to their effective antimicrobial and good release properties. ${ }^{1-4}$ However, the toxicology of AgNPs remains largely unknown. ${ }^{5,6}$ Moreover, AgNPs can be absorbed into the bloodstream via different routes of administration, leading to deposition of silver in many organs, including the liver and spleen, and potential organ damage after a certain amount of accumulation., ${ }^{1,7-9}$ Therefore, in the actual 
application process, protecting their antibacterial properties while reducing their absorption and toxicity in the body has become a very important challenge.

Previous studies have shown that different surface stabilizers have distinct impacts on AgNP cytotoxicity. Because of its good biocompatibility and antibacterial properties, chitosan is often used as the active ingredient of topical wound materials. ${ }^{10}$ It has been reported that chitosan is a good stabilizer for AgNPs and promotes wound healing by AgNPs. ${ }^{11}$ Moreover, chitosan-coated AgNPs demonstrated high efficiency in killing common gram-positive and -negative bacteria, and fungi. ${ }^{12,13}$ Compared with a higher degree of polymerization of chitosan, low molecular weight chitosan (LMWC) has better water solubility and biological activity, ${ }^{14}$ suggesting that LMWC may be an ideal stabilizer of AgNPs.

Methicillin-resistant Staphylococcus aureus (MRSA) infection is the most common clinical drug-resistant infection in the burn ward, with MRSA wound infection rate accounting for more than $60 \%$; moreover, the number of different MRSA strains isolated sharply increases and there is a growing number of multidrug-resistant MRSA. ${ }^{15}$ Thus, control of MRSA wound infection has become a global health problem. A previous study has shown that AgNPs have a great anti-bactericidal activity against MRSA. ${ }^{16}$ In order to obtain more safe and effective AgNP products for the clinical management of MRSA infection, in this study, we used LMWC as a reducing agent and a stabilizer to synthesize AgNPs, and compared its effects on the antibacterial activity, biocompatibility, and body absorption of AgNPs with different surface stabilizers of the same particle size.

\section{Materials and methods}

\section{Synthesis of LMWC}

Medical grade chitosan with 95\% degree of deacetylation was provided by Oriental Medical Institute (Qidong, Jiangsu, People's Republic of China). Three grams of chitosan was dissolved in $100 \mathrm{~mL} \mathrm{3 \%}$ acetic acid solution, followed by addition of $60 \mathrm{~mL} 30 \% \mathrm{H}_{2} \mathrm{O}_{2}$ and supplemented with $300 \mathrm{~mL}$ of deionized water. After incubation at $50^{\circ} \mathrm{C}$ in a water bath for 2 hours, the reaction was cooled in ice water, adjusted to $\mathrm{pH} 7.0$ with $2 \mathrm{M} \mathrm{NaOH}$, and filtered. The LMWC was precipitated with $900-1,500 \mathrm{~mL}$ of $100 \%$ ethanol and dried by vacuum. The molecular weight and traits of synthesized LMWC were determined by high-performance liquid chromatography (Waters 1525, UK) and Fourier transform infrared spectroscopy (FTIR, Nicolet 6700; Thermo Fisher Scientific, Waltham, MA, USA), respectively.

\section{Synthesis of low molecular weight chitosan-coated silver nanoparticles}

Four grams of LMWC was mixed with $0.1 \mathrm{~g} \mathrm{AgNO}_{3}$ in $50 \mathrm{~mL}$ deionized water at $50^{\circ} \mathrm{C}$ under stirring at $200 \mathrm{rpm}$ overnight in a hood. On the next day, acetone was added slowly with stirring until the solution became cloudy, followed by centrifugation at 2,000 rpm for 30 minutes. The supernatant was transferred to a new tube, dried by vacuum, and dissolved in 2-3 mL deionized water. The concentration of the resulting low molecular weight chitosan-coated silver nanoparticles (LMWC-AgNPs) was determined by UV-Vis (UV3600; Shimadzu Corporation, Kyoto, Japan) and ICP-MS (Agilent Technologies, Richardson, TX, USA). The particle size of LMWC-AgNPs was determined by transmission electron microscopy (TEM) (JEM-100S; JEOL, Tokyo, Japan).

\section{Synthesis of polyvinylpyrrolidone-coated silver nanoparticles}

One gram of polyvinylpyrrolidone (MW58000; Aladdin, People's Republic of China) was completely dissolved in $25 \mathrm{~mL}$ of absolute ethanol at $80^{\circ} \mathrm{C}$ for 1 hour, followed by addition of $0.2 \mathrm{~g} \mathrm{AgNO}_{3}$. The reaction continued under stirring $(400 \mathrm{rpm})$ until the brown color became stable (about 1-2 hours). Then, a total volume of 5-10 fold of acetone was added slowly with stirring until the solution became cloudy, followed by centrifugation at 2,000 rpm for 30 minutes. The supernatant was transferred to a new tube, dried by vacuum, and dissolved in 2-3 $\mathrm{mL}$ of deionized water. The concentration of the resulting polyvinylpyrrolidonecoated silver nanoparticles (PVP-AgNPs) was determined by UV-Vis and ICP-MS. The particle size of PVP-AgNPs was determined by TEM.

\section{Cell proliferation assay}

Silver nanoparticles without surface stabilizer (uncoatedAgNPs) were purchased from Shanghai Nanotechnology Ltd (Shanghai, People's Republic of China). Human fibroblast HS27 cells were from Shanghai Bioleaf Biotech (Shanghai, People's Republic of China) and cultured in Dulbecco's Modified Eagle's Medium supplemented with $10 \%$ fetal bovine serum, $100 \mathrm{U} / \mathrm{ml}$ penicillin, and $100 \mu \mathrm{g} / \mathrm{mL}$ streptomycin under a humidified $5 \% \mathrm{CO}_{2}$ atmosphere at $37^{\circ} \mathrm{C}$ in an incubator. Cells were seeded in triplicates in 96-well plates ( $1 \times 10^{4}$ cells in $100 \mu \mathrm{L}$ medium per well). After 24 hours incubation, the cells were added to $10 \mu \mathrm{L}$ of different AgNPs. After 24 hours, the cell counting kit-8 (CCK-8) assay was performed according to the manufacturer's recommendations. The absorbency was measured with Infinite M200 Pro 
multifunctional microplate reader (Tecan, Männedorf, Switzerland) at a wavelength of $450 \mathrm{~nm}$. All assays were performed in triplicates.

\section{Detection of cell apoptosis assay by Hoechst/propidium iodide staining}

Human fibroblast HS27 cells were cultured to $80 \%$ confluence and treated with $30 \mu \mathrm{g} / \mathrm{mL}$ of three types of AgNPs for 24 hours. After washing three times with phosphatebuffered saline, the cells were added to $10 \mu \mathrm{L}$ Hoechst 33258 $(5 \mu \mathrm{g} / \mathrm{mL})$ and incubated at $37^{\circ} \mathrm{C}$ for 10 minutes, followed by adding $5 \mu \mathrm{L}$ PI $(15 \mu \mathrm{g} / \mathrm{mL})$ in the dark at room temperature for 10 minutes. The cells were observed under a fluorescence microscope (Ti-S; Nikon, Minato, Japan).

\section{In vitro bacterial killing}

A bioluminescent MRSA strain derived from ATCC 33591 was a gift from Michael Hamblin's lab (Wellman Center for Photomedicine, MGH, Boston, MA, USA). In total, $0.5 \mathrm{~mL}$ of logarithmic growth phase MRSA $\left(1.5 \times 10^{8} \mathrm{cfu} / \mathrm{mL}\right)$ suspension was mixed with $0.5 \mathrm{~mL}$ organic interfering substances ( $3 \%$ bovine serum albumin) and incubated in a $20^{\circ} \mathrm{C} \pm 1^{\circ} \mathrm{C}$ water bath for 5 minutes. The bacterial solution was added with $4.0 \mathrm{~mL}$ of different concentrations of three types of AgNPs and incubated for 30 minutes. Also, $0.5 \mathrm{~mL}$ of the mixture was mixed with $4.5 \mathrm{~mL}$ of sterile saline and incubated for 10 minutes at room temperature. In total, $100 \mu \mathrm{L}$ of the sample solution was coated on a flat plate, incubated overnight at $35^{\circ} \mathrm{C}$, and the number of bacterial colonies was calculated. For filter paper assay (bacterial killing measurement), $20 \mu \mathrm{L}$ of $10 \mu \mathrm{g} / \mathrm{mL}$ AgNP solution was dropped onto a $6 \mathrm{~mm}$ sterile filter paper, dried at $37^{\circ} \mathrm{C}$, and placed onto a dish densely coated with logarithmic growth phase MRSA $\left(1.5 \times 10^{8} \mathrm{cfu} / \mathrm{mL}\right)$ suspension with a sterile cotton swab. The dish containing MRSA and AgNP-filter paper was placed at $35^{\circ} \mathrm{C} 5 \% \mathrm{CO}_{2}$ incubator and inhibition zone size was observed 48 hours later.

\section{Animal experiments}

The Animal Committee of Ninth People's Hospital Affiliated to Shanghai Jiao Tong University, School of Medicine approved all the experimental protocols and animal handling procedures. All experimental procedures and postoperative animal care were conducted in accordance with the National Institute of Health's Guidelines for the Care and Use of Laboratory Animals. Balb/c mice were purchased from SLAC Laboratory Animal Technology (Shanghai, People's Republic of China). All mice were maintained on a 12-hour light/dark cycle in a room at $22^{\circ} \mathrm{C}-25^{\circ} \mathrm{C}$ and allowed free access to food and water before surgery. Balb/c mice with $1.5 \times 1.5 \mathrm{~cm}$ back full-thickness skin wounds were inoculated with $100 \mu \mathrm{L}$ of $9 \times 10^{8} \mathrm{cfu} / \mathrm{mL}$ MRSA bacteria, and infection of mice was assessed 3 days later with Fx CCD luminescent camera (Vilber Lourmat, Collégien, France). The mice were randomly divided into groups. The infected wounds were dressed with $50 \mu \mathrm{g} / \mathrm{g} \cdot \mathrm{bw}$ of different AgNPs, bandaged with double gauze bandage, and fixed with $3 \mathrm{M}$ film. In the following 2 consecutive days, Fx CCD luminescent camera was used to observe wound infection and healing. The dressing contained AgNPs was changed every other day. On day 14, fresh blood was taken from mouse eyeballs and centrifuged at $1,500 \mathrm{rpm}$ for 10 minutes. The supernatant sera were subjected to liver function analysis with DXC800 Beckman automatic biochemical analyzer (Beckman Coulter, USA). At the same time, a portion of liver tissue and healed skin tissue on the wounded area was removed, dissolved in 69\% concentrated nitric acid solution, and subjected to silver content determination by ICP-MS in order to assess the body's absorption of AgNPs.

\section{Statistical analyses}

Graphs were constructed using GraphPad Prism (GraphPad Software, San Diego, CA, USA). All data are presented as mean \pm standard error of mean. Statistical significance was determined by unpaired two-tailed $t$-tests or two-way analysis of variance. $P<0.05$ was considered statistically significant.

\section{Results \\ The characteristics of LMWC-AgNPs, PVP-AgNPs, and uncoated-AgNPs}

FTIR tests showed that the depolymerization during the process of production of LMWC did not change the molecular structure of chitosan, with only molecular chain rupture, as demonstrated by similar FTIR spectra characteristic of LMWC and chitosan (Figure 1A). The molecular weight of LMWC was between 2,000 and 2,480 g/mol, mainly in the vicinity of 2,300 g/mol (Figure 1B). As the molecular weight of chitosan monomer is $161.2 \mathrm{~g} / \mathrm{mol}$, these results indicated that our synthesized LMWC was a chain of 12-15 chitosan sugar monomers. To compare the cytotoxicity of LMWC with chitosan, human fibroblast HS27 cells were treated with different concentrations of LMWC or chitosan $(200-1,000 \mu \mathrm{g} / \mathrm{mL})$ for 24 hours, and cell viability was assessed by CCK-8 assay. As shown in Figure 1C, LMWC was apparently less toxic to HS27 cells than chitosan. UV-Vis analysis demonstrated that absorption curves of 
A

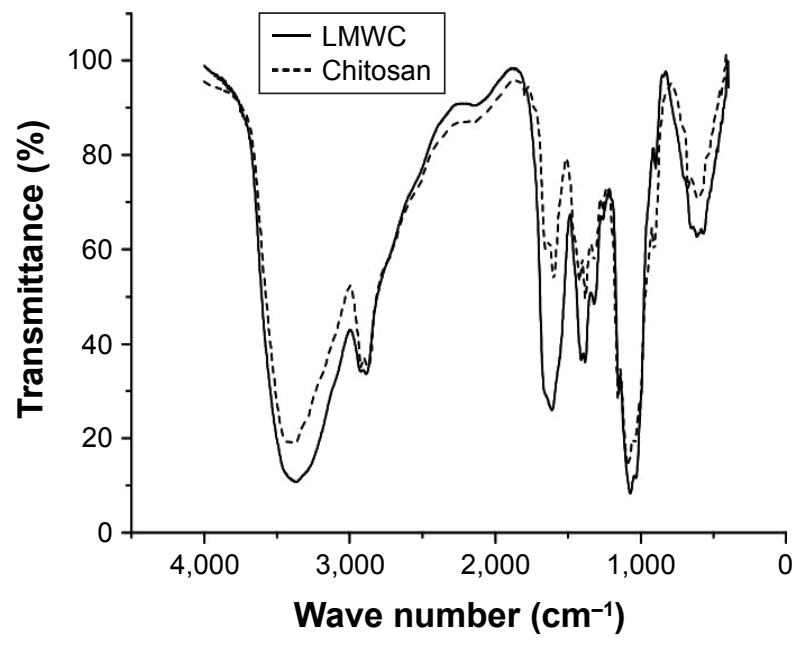

B

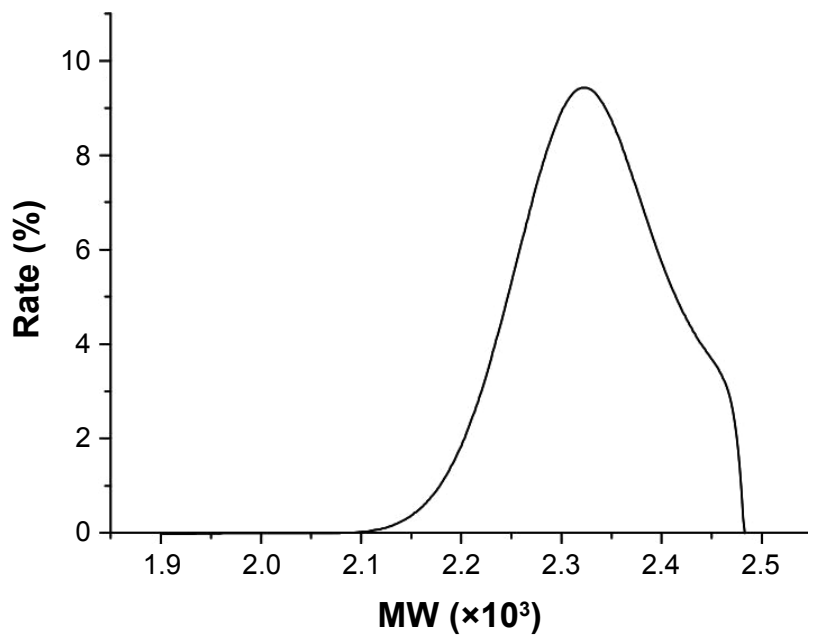

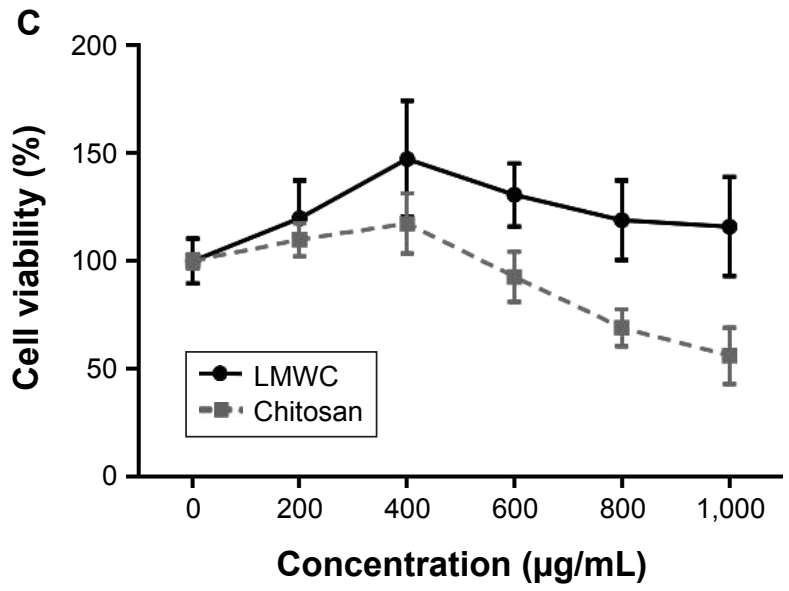

Figure I Characterization of molecular weight distribution of LMWC.

Notes: (A) FTIR spectra of chitosan (full line) and LMWC (short dash line) showed similar absorption. (B) FTIR analysis of molecular weight distribution of LMWC. (C) HS27 cells were treated with the indicated concentrations of LMWC or chitosan for 24 hours. Cell viability was assessed by CCK-8 assay.

Abbreviations: FTIR, Fourier transform infrared spectroscopy; LMWC, low-molecular weight chitosan; CCK-8, cell counting kit-8; MW, molecular weight.

LMWC-AgNPs, PVP-AgNPs, and uncoated-AgNPs were similar, with maximum absorption peaks at 414,408 , and $421 \mathrm{~nm}$, respectively (Figure 2). The particle size of LMWCAgNPs (Figure 3A), PVP-AgNPs (Figure 3C), and uncoatedAgNPs (Figure 3E) was detected by TEM. Further, ImageJ software analysis of the size and distribution of AgNPs of LMWC-AgNPs (Figure 3B), PVP-AgNPs (Figure 3D), and uncoated-AgNPs (Figure 3F) showed that their sizes were in the range of 10-30 $\mathrm{nm}$ without significant difference.

\section{LMWC-AgNPs, PVP-AgNPs, and} uncoated-AgNPs have similar bactericidal effects

The anti-bactericidal effects of the three types of AgNPs were similar, with an IC50 of approximately $4 \mu \mathrm{g} / \mathrm{mL}$ for all the

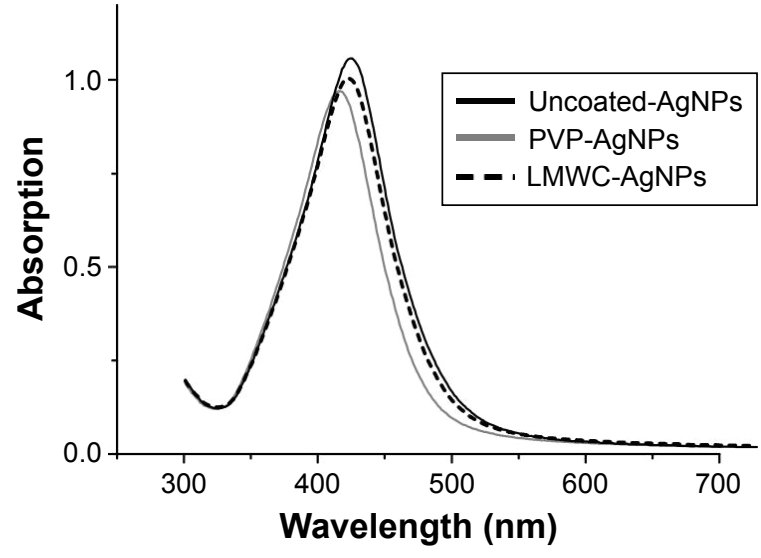

Figure 2 UV-Vis absorption spectra of uncoated-AgNPs (black full line), PVP-AgNPs (gray full line), and LMWC-AgNPs (black dash line).

Abbreviations: Uncoated-AgNPs, silver nanoparticles without surface stabilizer; PVP-AgNPs, polyvinylpyrrolidone-coated silver nanoparticles; LMWC-AgNPs, low molecular weight chitosan-coated silver nanoparticles. 
A
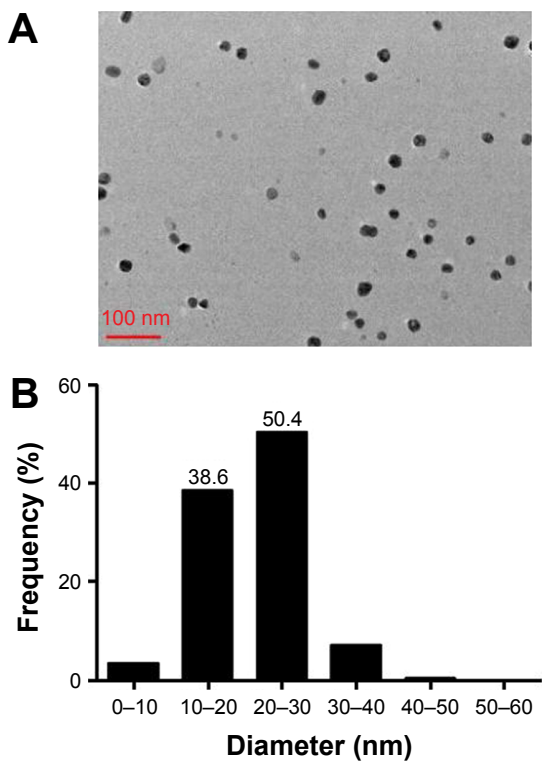

\section{C}
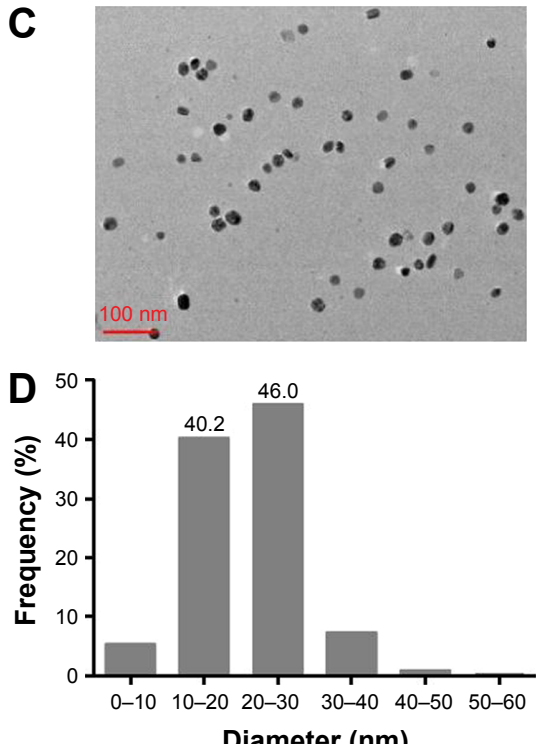

$\mathbf{E}$
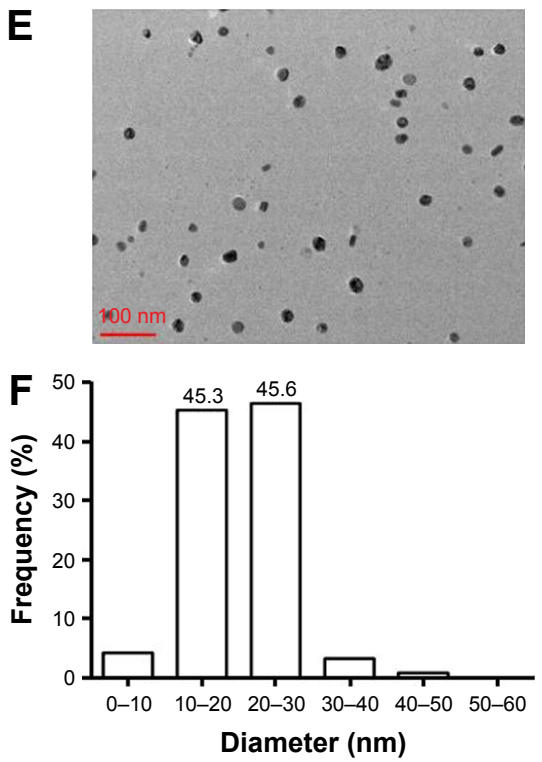

Figure 3 TEM analysis of the shape and size of nanoparticles. (A) TEM images of LMWC-AgNPs. (B) Imagej software analysis of the size and distribution of LMWC-AgNPs. (C) TEM images of PVP-AgNPs. (D) Imagej software analysis of the size and distribution of PVP-AgNPs. (E) TEM images of uncoated-AgNPs. (F) ImageJ software analysis of the size and distribution of uncoated-AgNPs.

Abbreviations: TEM, transmission electron microscopy; LMWC-AgNPs, low molecular weight chitosan-coated silver nanoparticles; PVP-AgNPs, polyvinylpyrrolidonecoated silver nanoparticles; uncoated-AgNPs, silver nanoparticles without surface stabilizer.

three types. At $8 \mu \mathrm{g} / \mathrm{mL}$, the anti-bactericidal effect reached $100 \%$, and there was no growth of bacteria following incubation with $10 \mu \mathrm{g} / \mathrm{mL}$ of any of the three types of AgNPs for 30 minutes (Figure 4A and B). Similar bactericidal effects of the three types of AgNPs were observed by bacteriostatic ring method (Figure 4C). In vivo bactericidal results showed that, on the third day, the amounts of bacteria in wounds on the back of mice were similar in the three types of AgNPs treatments, which were demonstrated by the similar values of MRSA chemiluminescent in the three groups (Figure 4D).

\section{LMWC-AgNPs are less toxic to human fibroblasts than PVP-AgNPs and uncoated-AgNPs}

To test the cytotoxicity of three types of AgNPs on human fibroblasts, HS27 cells were treated with different concentrations of the three types of AgNPs for 24 hours and cell viability was assessed by CCK-8 assay. As shown in Figure 5A, the cell viability of HS27 cells treated with 10 and $20 \mu \mathrm{g} / \mathrm{mL}$ of uncoated-AgNPs or PVP-AgNPs was $98.83 \% \pm 7.82 \%$, $86.47 \pm 16.92 \%$ or $99.84 \% \pm 7.82 \%, 89.47 \% \pm 13.92 \%$, respectively. Uncoated-AgNPs and PVP-AgNPs at $30 \mu \mathrm{g} / \mathrm{mL}$ were obviously toxic to cells with cell viability decreased to $62.75 \% \pm 14.75 \%$ and $68.64 \% \pm 11.03 \%$, respectively. At $50 \mu \mathrm{g} / \mathrm{mL}$ of either uncoated-AgNPs or PVP-AgNPs, the majority of cells were dead and floating with cell viability of only $16.08 \% \pm 4.05 \%$ and $13.12 \% \pm 3.75 \%$, respectively.
However, the cell viability of cells treated with 10, 20, 30, 40 , and $50 \mu \mathrm{g} / \mathrm{mL}$ of LMWC-AgNPs was $109.84 \% \pm 7.82 \%$, $107.46 \% \pm 13.92 \%, 96.75 \% \pm 11.65 \%, 85.08 \% \pm 8.55 \%$, and $66.08 \% \pm 13.05 \%$, respectively. There were no statistical differences in cell viability among the three types of AgNP treatments with lower concentrations (below $20 \mu \mathrm{g} / \mathrm{mL}$ ). Detection of apoptosis by Hoechst/PI staining of cells treated with $30 \mu \mathrm{g} / \mathrm{mL}$ AgNPs for 24 hours showed similar apoptosis rates for uncoated-AgNPs and PVP-AgNPs, while lower apoptosis rate for LMWC-AgNPs when compared with uncoated-AgNPs and PVP-AgNPs (Figure 5B). These results indicated that LMWC-AgNPs were less toxic to human fibroblasts cells compared to uncoated-AgNPs and PVP-AgNPs.

\section{LMWC-AgNPs, PVP-AgNPs, and uncoated-AgNPs effectively control MRSA wound infection and promote wound healing without any apparent difference}

Topical wound healing rate showed that, in comparison to the untreated control group, each group treated with AgNPs displayed accelerated wound healing on day 3 (Figure 6A and B). On day 14, most wounds healed in all the groups treated with AgNPs (Figure 6A and B). Thus, the three types of AgNPs effectively controlled MRSA wound infection and promoted wound healing without apparent difference. 


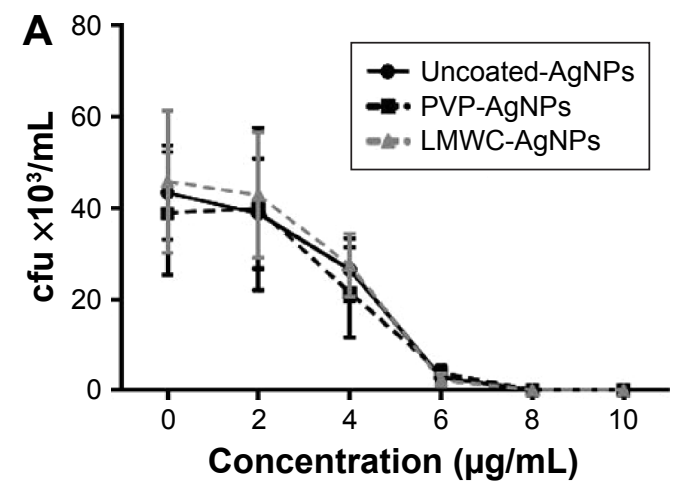

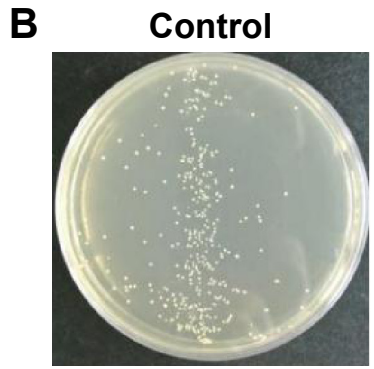
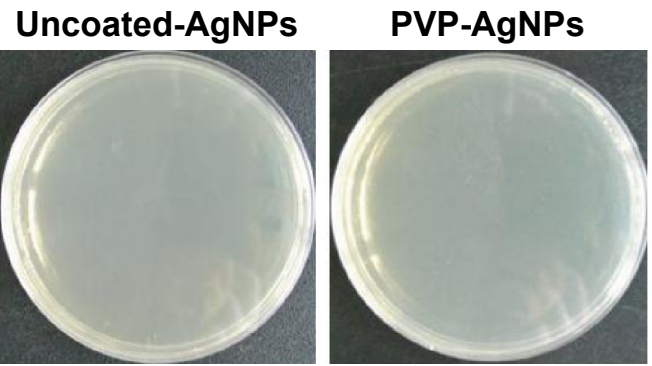

\section{LMWC-AgNPs}

C
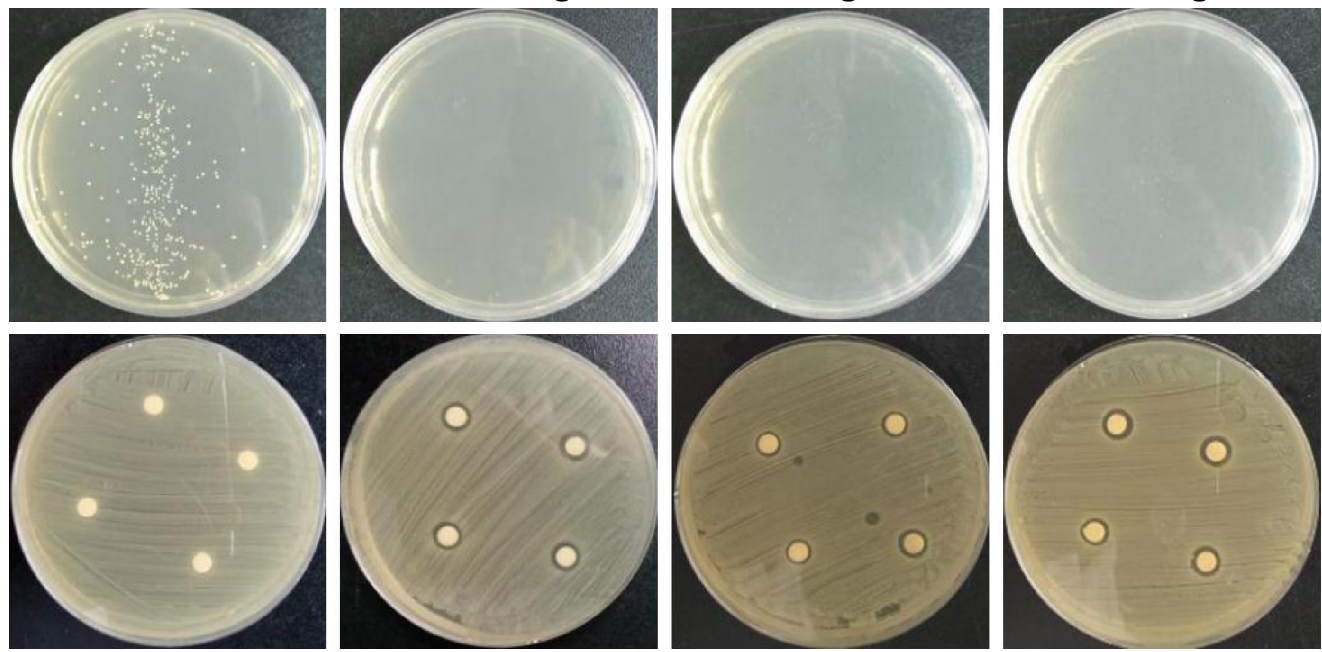

D

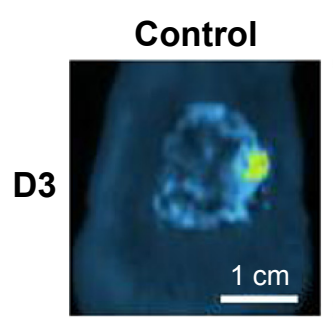

UncoatedAgNPs

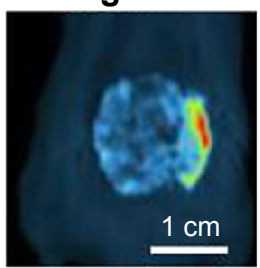

PVP-AgNPs

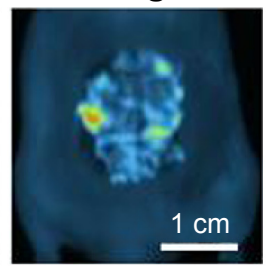

LMWC-
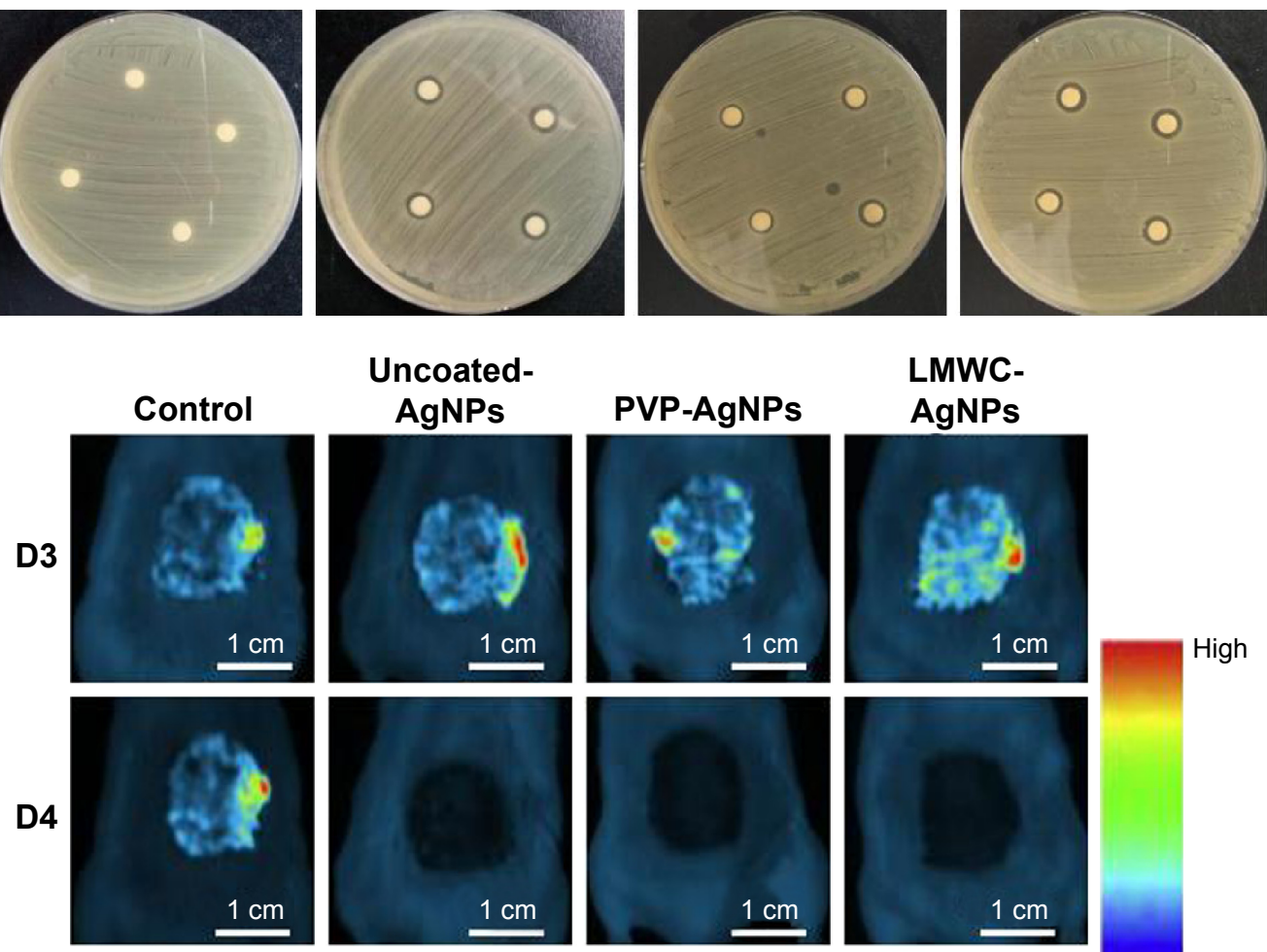

AgNPs
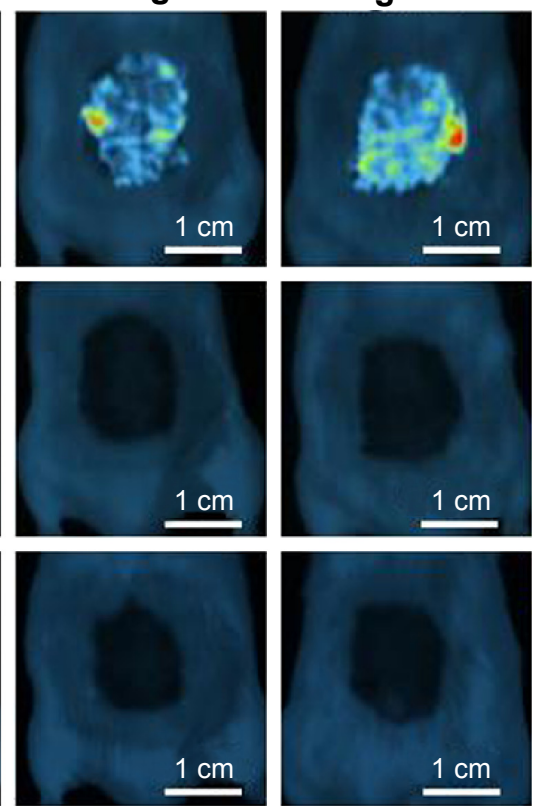

D5
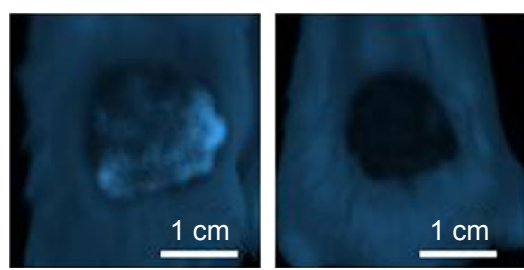

Figure 4 The anti-bactericidal effects of three types of AgNPs. (A) A bioluminescent MRSA strain was treated with the indicated concentrations of the three types of AgNPs for 48 hours. The viability of MRSA was calculated by RFU. (B) A set of representative photos of three independent experiments of (A) with I0 $\mu g / m L$ of AgNPs. (C) Analysis of bactericidal effects of the three types of AgNPs by bacteriostatic ring method. A set of representative photos of three independent experiments with $10 \mu g / m L$ of $A g N P s$ is shown. (D) Mice wounds were inoculated with MRSA and dressed with different AgNPs. From day 3 to day 5, the amounts of bacteria in wounds on the backs of mice were determined by FX CCD chemiluminescence assay. Higher intensity of the red color indicates more severe infection. Scale bar means I cm.

Abbreviations: AgNPs, silver nanoparticles; MRSA, methicillin-resistant Staphylococcus aureus; RFU, relative fluorescence units; Uncoated-AgNPs, silver nanoparticles without surface stabilizer; PVP-AgNPs, polyvinylpyrrolidone-coated silver nanoparticles; LMWC-AgNPs, low molecular weight chitosan-coated silver nanoparticles; D, day. 


\section{A}

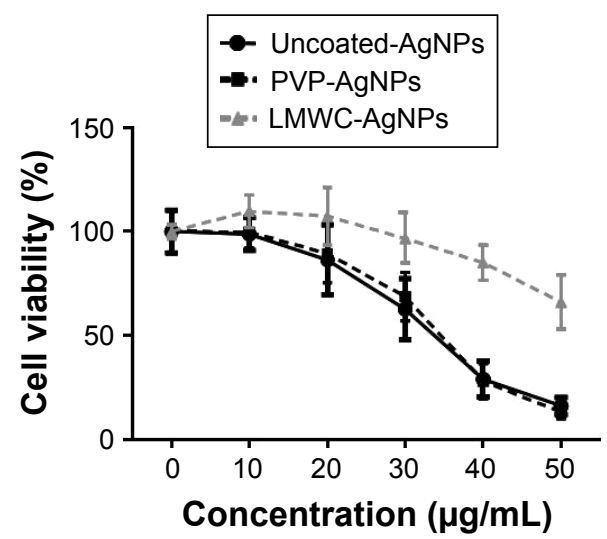

B
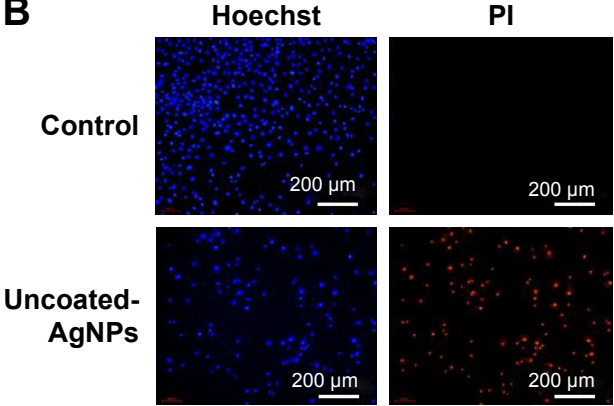

PVP.
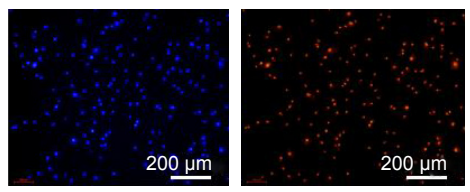

LMWC-
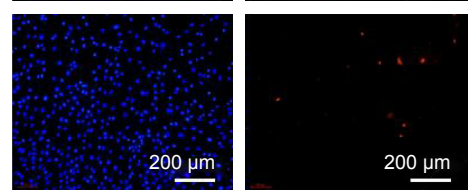

Figure $\mathbf{5}$ The cytotoxicity of the three types of AgNPs on human fibroblast HS27 cells. (A) HS27 cells were treated with the indicated concentrations of the three types of AgNPs for 24 hours. Cell viability was assessed by CCK-8 assay. (B) HS27 cells were treated with $30 \mu g / m L$ of the the three types of AgNPs for 24 hours. Cell apoptosis was assessed by Hoechst/PI staining, Scale bar means $200 \mu \mathrm{m}$.

Abbreviations: AgNPs, silver nanoparticles; CCK-8, cell counting kit-8; PI, propidium iodide; Uncoated-AgNPs, silver nanoparticles without surface stabilizer; PVP-AgNPs, polyvinylpyrrolidone-coated silver nanoparticles; LMWC-AgNPs, low molecular weight chitosan-coated silver nanoparticles.
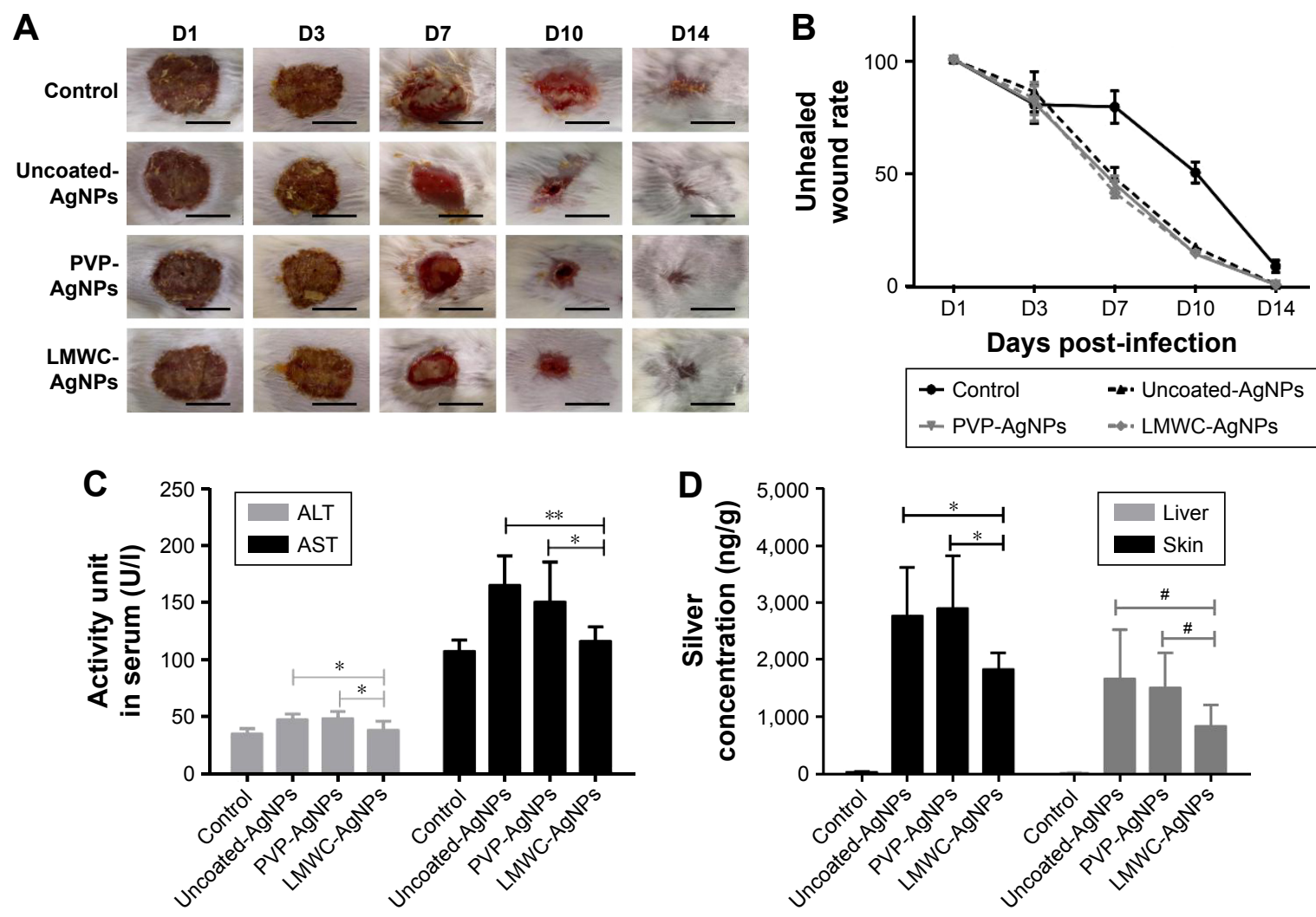

Figure 6 The effects of LMWC-AgNPs, PVP-AgNPs, and uncoated-AgNPs on the control of MRSA wound infection and wound healing in mice. (A) Fx CCD luminescent camera images of wound infection and healing following dressing with different AgNPs for the indicated days. Scale bar means I cm. (B) Quantitation of the topical wound healing rate. (C) After application of AgNPs for II days, the ALT and AST levels in the blood of mice were detected for liver function. After applying AgNPs six times over II days, the ALT levels in the blood of mice were detected for liver function. $* P<0.05 ; * * P<0.01$. (D) Mouse liver tissues and healed skin tissue at wounded area were removed after continuous application of AgNPs, on day 14, and subjected to silver content determination by ICP-MS. ${ }^{* P}<0.05$ in liver; ${ }^{*}<<0.05$ in skin.

Abbreviations: LMWC-AgNPs, low molecular weight chitosan-coated silver nanoparticles; PVP-AgNPs, polyvinylpyrrolidone-coated silver nanoparticles; uncoated-AgNPs, silver nanoparticles without surface stabilizer; MRSA, methicillin-resistant Staphylococcus aureus; AgNPs, silver nanoparticles; ALT, alanine aminotransferase; AST, aspartate aminotransferase; D, day; ICP-MS, inductively coupled plasma mass spectrometry. 
LMWC-AgNPs-treated mice have significantly reduced liver deposition of silver in comparison to mice treated with uncoated-AgNPs or PVP-AgNPs

After six times of application of AgNPs for 11 days (D3/D5/ D7/D9/D11/D13), the blood of mice was analysed for liver function by assessing alanine aminotransferase (ALT) and aspartate aminotransferase (AST) levels. The ALT values of mice in the uncoated-AgNP and PVP-AgNP groups were $47.33 \pm 4.95$ and $48.30 \pm 6.18 \mathrm{U} / \mathrm{L}$, respectively, while the ALT value of mice in the LMWC-AgNP group was $38.00 \pm 8.03 \mathrm{U} / \mathrm{L}$, which was significantly lower than the uncoated-AgNP and PVP-AgNP groups $(P<0.05)$. Similar results were obtained for AST values (Figure 6C). In order to assess the body's absorption of AgNPs, mouse liver tissues and healed skin tissue at the wounded area were removed on day 14 after continuous application of AgNPs on day 14, dissolved in $69 \%$ concentrated nitric acid solution, and subjected to silver content determination by ICP-MS. The liver silver content in the mice treated with uncoated-AgNPs and PVP-AgNPs was $2,765.85 \pm 853.49$ and $2,898.48 \pm 918.96 \mathrm{ng} / \mathrm{g}$, respectively, while that in the mice treated with LMWC-AgNPs was $1,827.97 \pm 294.16 \mathrm{ng} / \mathrm{g}$, which was significantly lower than the uncoated-AgNP and PVP-AgNP groups $(P<0.05$, Figure 6D). Further analysis of the silver content of the newly healed skin tissues revealed that the silver content in the newly healed skin tissues of the mice treated with uncoated-AgNPs

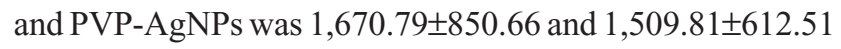
$\mathrm{ng} / \mathrm{g}$, respectively, while the silver content in the mice treated with LMWC-AgNPs was $837.35 \pm 371.36 \mathrm{ng} / \mathrm{g}$, which was significantly lower than the uncoated-AgNP and PVP-AgNP groups ( $P<0.05$, Figure 6D).

\section{Discussion}

Due to their effective antibacterial property, AgNPs are widely used as various types of medical materials including trauma topical material. ${ }^{4,17,18}$ A large number of previous studies have shown that, compared with the silver ions, the cytotoxicity of AgNPs has been greatly decreased; however, AgNPs still induce cell damage through induction of apoptosis. ${ }^{19-21}$ The cytotoxicity of AgNPs could be influenced by particle size, surface-stabilizing agents, $\mathrm{pH}$, and some other factors. ${ }^{1,5,7,22-24}$ The surface-stabilizing coating is one of the most important factors for protecting cell viability from AgNPs. ${ }^{25-27}$

Previous studies have shown that chitosan has a very good biocompatibility and antibacterial properties. In addition, AgNPs/chitosan materials could promote wound healing. ${ }^{3,17,28-30}$ In comparison with long-chain high molecular weight chitosan, the short-chain LMWC is better in terms of water solubility and biocompatibility. Besides, it has been confirmed that LMWC enhances the physical and chemical stability of many drugs such as insulin ${ }^{31}$ and siRNA, ${ }^{32}$ and works as a suitable carrier for the delivery and controlled release of drugs. In this study, we used hydrogen peroxide method to decompose high molecular weight chitosan and obtained short-chain molecules of chitosan with molecular weight between 2,000 and 2,500 Da. This short-chain molecule had a good solubility in water, thus avoiding the requirement of an acidic solution to dissolve high molecular weight chitosan. Compared to high molecular weight chitosan, this LMWC demonstrated a better biological activity (Figure 1C). The obtained LMWC was used as a stabilizer and a reducing agent for direct synthesis of LMWC-AgNPs with particle diameter size of 10-30 nm. In the current study, the three types of AgNPs were 10-30 $\mathrm{nm}$ with the same distribution but different coatings. All the three types of AgNPs caused no obvious cytotoxicity at lower concentrations ( 10 and $20 \mu \mathrm{g} / \mathrm{mL}$ ). When the concentration increased to up to $30 \mu \mathrm{g} / \mathrm{mL}$, however, treatment with LMWC-AgNPs led to significant less toxicity compared with the other two types of AgNP treatments, in the viability of human fibroblast HS27 cells (Figure 5A). This indicates that the surface coating with LMWC-AgNPs reduces cell toxicity and increases cell viability, although further study is needed for more detailed explanation. In comparison to PVP-AgNPs and uncoated-AgNPs, LMWC-AgNPs had similar effects of killing MRSA effects but were less toxic to human fibroblasts. Mice experiments also showed that the three types of AgNPs had good anti-inflammatory effects and effective protection of wound healing in mice.

Previous studies have shown that, through various means such as oral intake, intraperitoneal injection, and intravenous injection, AgNPs can be distributed and deposited on various organs of the body, with the deposition mainly in the liver and spleen. ${ }^{1,79,33,34}$ In addition, it has been reported that the ALT and AST levels in mice serum rose significantly after 2 days of oral intake of $20 \mu \mathrm{g} / \mathrm{g}$ AgNPs with a size of 35-45 nm. ${ }^{9}$ Intriguingly, in the current study, we found that after 11 days of application, the liver silver content in the mice treated with LMWC-AgNPs was significantly lower than that in the mice treated with uncoated-AgNPs or PVP-AgNPs. This suggests less LMWC-AgNP absorption into the wound and organs than with uncoated-AgNPs or PVP-AgNPs. Similarly, the silver content of the newly healed skin tissues of the mice treated with LMWC-AgNPs 
was significantly lower than that in the uncoated-AgNP and PVP-AgNP groups. Furthermore, both the ALT and AST levels in mice serum in the LMWC-AgNP-treated group were much less than those in the uncoated-AgNP-treated or PVP-AgNP-treated groups. Our results suggest that LMWCAgNPs have the advantage of less body absorption while remain the anti-infection properties.

In summary, by application of an LMWC as a stabilizer and a reducing agent, we synthesized a diameter of 10-30 nm LMWC-AgNPs. We reveal for the first time that LMWC-AgNPs the characteristics of anti-MRSA infection, low cytotoxicity, better biocompatibility, and lower body absorption in both in vitro and in vivo experiments, when compared with PVP-AgNP and uncoated-AgNP treatments. Our study suggests that LMWC-AgNPs have less risk of damage to organ functions and are effective and safer AgNPs for medical materials, which is very important for large area burn patients, who need local usage of antibacterial materials on wounds for a long time after injury.

\section{Acknowledgment}

This research was supported by the Youth Program of National Natural Science Fund Projects (81501656).

\section{Disclosure}

The authors report no conflicts of interest in this work.

\section{References}

1. Ahamed M, Alsalhi MS, Siddiqui MK. Silver nanoparticle applications and human health. Clin Chim Acta. 2010;411(23-24):1841-1848.

2. Rennukka M, Sipaut CS, Amirul AA. Synthesis of poly(3hydroxybutyrate-co-4-hydroxybutyrate)/chitosan/silver nanocomposite material with enhanced antimicrobial activity. Biotechnol Prog. 2014; 30(6): 1469-1479.

3. Archana D, Singh BK, Dutta J, Dutta PK. Chitosan-PVP-nano silver oxide wound dressing: in vitro and in vivo evaluation. Int $J$ Biol Macromol. 2015;73:49-57.

4. Rath G, Hussain T, Chauhan G, Garg T, Goyal AK. Collagen nanofiber containing silver nanoparticles for improved wound-healing applications. $J$ Drug Target. 2016;24(6):520-529.

5. de Lima R, Seabra AB, Duran N. Silver nanoparticles: a brief review of cytotoxicity and genotoxicity of chemically and biogenically synthesized nanoparticles. J Appl Toxicol. 2012;32(11):867-879.

6. Beer C, Foldbjerg R, Hayashi Y, Sutherland DS, Autrup H. Toxicity of silver nanoparticles - nanoparticle or silver ion? Toxicol Lett. 2012; 208(3):286-292.

7. Bergin IL, Wilding LA, Morishita M, et al. Effects of particle size and coating on toxicologic parameters, fecal elimination kinetics and tissue distribution of acutely ingested silver nanoparticles in a mouse model. Nanotoxicology. 2016;10(3):352-360.

8. Lankveld DP, Oomen AG, Krystek P, et al. The kinetics of the tissue distribution of silver nanoparticles of different sizes. Biomaterials. 2010; 31(32):8350-8361.

9. Heydrnejad MS, Samani RJ, Aghaeivanda S. Toxic effects of silver nanoparticles on liver and some hematological parameters in male and female mice (Mus musculus). Biol Trace Elem Res. 2015;165(2):153-158.
10. Ishihara $\mathrm{M}$, Nguyen VQ, Mori $\mathrm{Y}$, Nakamura $\mathrm{S}$, Hattori H. Adsorption of silver nanoparticles onto different surface structures of chitin/ chitosan and correlations with antimicrobial activities. Int J Mol Sci. 2015;16(6):13973-13988.

11. Li CW, Wang Q, Li J, et al. Silver nanoparticles/chitosan oligosaccharide/poly(vinyl alcohol) nanofiber promotes wound healing by activating TGFbeta1/Smad signaling pathway. Int J Nanomedicine. 2016;11:373-386

12. Wei D, Sun W, Qian W, Ye Y, Ma X. The synthesis of chitosan-based silver nanoparticles and their antibacterial activity. Carbohydr Res. 2009;344(17):2375-2382.

13. Wang LS, Wang CY, Yang CH, et al. Synthesis and anti-fungal effect of silver nanoparticles-chitosan composite particles. Int J Nanomedicine. 2015;10:2685-2696.

14. Richardson SC, Kolbe HV, Duncan R. Potential of low molecular mass chitosan as a DNA delivery system: biocompatibility, body distribution and ability to complex and protect DNA. Int J Pharm. 1999; 178(2):231-243.

15. Branski LK, Al-Mousawi A, Rivero H, Jeschke MG, Sanford AP, Herndon DN. Emerging infections in burns. Surg Infect (Larchmt). 2009;10(5):389-397.

16. Ansari MA, Khan HM, Khan AA, Cameotra SS, Alzohairy MA. Antibiofilm efficacy of silver nanoparticles against MRSA and MRSE isolated from wounds in a tertiary care hospital. Indian J Med Microbiol. 2015;33(1):101-109.

17. Levi-Polyachenko N, Jacob R, Day C, Kuthirummal N. Chitosan wound dressing with hexagonal silver nanoparticles for hyperthermia and enhanced delivery of small molecules. Colloids Surf B Biointerfaces. 2016;142:315-324.

18. GhavamiNejad A, Park CH, Kim CS. In situ synthesis of antimicrobial silver nanoparticles within antifouling zwitterionic hydrogels by catecholic redox chemistry for wound healing application. Biomacromolecules. 2016;17(3):1213-1223.

19. Arai Y, Miyayama T, Hirano S. Difference in the toxicity mechanism between ion and nanoparticle forms of silver in the mouse lung and in macrophages. Toxicology. 2015;328:84-92.

20. Vannini C, Domingo G, Onelli E, et al. Morphological and proteomic responses of Eruca sativa exposed to silver nanoparticles or silver nitrate. PLoS One. 2013;8(7):e68752.

21. Sun C, Yin N, Wen R, et al. Silver nanoparticles induced neurotoxicity through oxidative stress in rat cerebral astrocytes is distinct from the effects of silver ions. Neurotoxicology. 2016;52:210-221.

22. Dhawan A, Sharma V. Toxicity assessment of nanomaterials: methods and challenges. Anal Bioanal Chem. 2010;398(2):589-605.

23. Park MV, Neigh AM, Vermeulen JP, et al. The effect of particle size on the cytotoxicity, inflammation, developmental toxicity and genotoxicity of silver nanoparticles. Biomaterials. 2011;32(36):9810-9817.

24. Osborne OJ, Lin S, Chang CH, et al. Organ-specific and size-dependent Ag nanoparticle toxicity in gills and intestines of adult zebrafish. ACS Nano. 2015;9(10):9573-9584.

25. Zhang XF, Liu ZG, Shen W, Gurunathan S. Silver nanoparticles: synthesis, characterization, properties, applications, and therapeutic approaches. Int J Mol Sci. 2016;17(9):1534.

26. Wei L, Lu J, Xu H, Patel A, Chen ZS, Chen G. Silver nanoparticles: synthesis, properties, and therapeutic applications. Drug Discov Today. 2015;20(5):595-601.

27. Zhang T, Wang L, Chen Q, Chen C. Cytotoxic potential of silver nanoparticles. Yonsei Med J. 2014;55(2):283-291.

28. Freire PL, Albuquerque AJ, Farias IA, et al. Antimicrobial and cytotoxicity evaluation of colloidal chitosan - silver nanoparticles - fluoride nanocomposites. Int J Biol Macromol. 2016;93(Pt A):896-903.

29. Salama HE, Saad GR, Sabaa MW. Synthesis, characterization, and biological activity of cross-linked chitosan biguanidine loaded with silver nanoparticles. J Biomater Sci Polym Ed. Epub 2016 Oct 2:1-19.

30. Verma J, Kanoujia J, Parashar P, Tripathi CB, Saraf SA. Wound healing applications of sericin/chitosan-capped silver nanoparticles incorporated hydrogel. Drug Deliv Transl Res. Epub 2016 Aug 26. 
31. Al-Kurdi ZI, Chowdhry BZ, Leharne SA, Al Omari MM, Badwan AA. Low molecular weight chitosan-insulin polyelectrolyte complex: characterization and stability studies. Mar Drugs. 2015;13(4):1765-1784.

32. Fernandes JC, Qiu X, Winnik FM, et al. Low molecular weight chitosan conjugated with folate for siRNA delivery in vitro: optimization studies. Int J Nanomedicine. 2012;7:5833-5845.
33. Takenaka S, Karg E, Roth C, et al. Pulmonary and systemic distribution of inhaled ultrafine silver particles in rats. Environ Health Perspect. 2001;109(Suppl 4):547-551.

34. Choi JE, Kim S, Ahn JH, et al. Induction of oxidative stress and apoptosis by silver nanoparticles in the liver of adult zebrafish. Aquat Toxicol. 2010;100(2):151-159.

\section{Publish your work in this journal}

The International Journal of Nanomedicine is an international, peerreviewed journal focusing on the application of nanotechnology in diagnostics, therapeutics, and drug delivery systems throughout the biomedical field. This journal is indexed on PubMed Central, MedLine, CAS, SciSearch ${ }^{\circledR}$, Current Contents ${ }^{\circledR} /$ Clinical Medicine,
Journal Citation Reports/Science Edition, EMBase, Scopus and the Elsevier Bibliographic databases. The manuscript management system is completely online and includes a very quick and fair peer-review system, which is all easy to use. Visit http://www.dovepress.com/ testimonials.php to read real quotes from published authors.

Submit your manuscript here: http://www.dovepress.com/international-journal-of-nanomedicine-journal 